\title{
Aspectos epidemiológicos, clínicos e anatomopatológicos da intoxicação por aceturato de diminazeno em cães $^{1}$
}

\author{
Mariana M. Flores ${ }^{2}$, Paula R. Pereira ${ }^{3}$, Alexandre Mazzanti ${ }^{4}$, Glaucia D. Kommers ${ }^{5}$ \\ e Rafael A. Fighera ${ }^{5 *}$
}

\begin{abstract}
Flores M.M., Pereira P.R., Mazzanti A., Kommers G.D. \& Fighera R.A. 2014. [Epidemiological, clinical, and pathological aspects of diminazene aceturate toxicosis in dogs.] Aspectos epidemiológicos, clínicos e anatomopatológicos da intoxicação por aceturato de diminazeno em cães. Pesquisa Veterinária Brasileira 34(7):667-674. Departamento de Patologia, Universidade Federal de Santa Maria, Av. Roraima 1000, Camobi, Santa Maria, RS 97105-900, Brazil. E-mail: anemiaveterinaria@yahoo.com.br

The epidemiological, clinical, and pathological aspects of diminazene aceturate (DA) spontaneous toxicosis were evaluated in 10 dogs. All affected dogs developed signs of thalamic-cortical syndrome, characterized mainly by neurological changes in the conscience levels, tetraparesis, extensor stiffness, and seizures. In some cases there was also evidence of cerebellar syndrome, characterized by generalized muscle tremors (high-frequency and low-amplitude) and/or vestibular syndrome, characterized by or ataxia, head tilt, and falling. These clinical signs occurred between 24 and 48 hours following intramuscular administration of DA and persisted until spontaneous death or euthanasia occurred between 1 and 7 days after the onset of clinical signs. The mentioned clinical signs reflected lesions that consisted of focal symmetrical hemorrhagic encephalomalacia affecting medulla oblongata, pons, cerebellar medulla, thalamus, midbrain, cerebellar peduncles, and basal nuclei. This article (1) describes and discusses DA toxicosis in dogs, a poorly-described clinical entity that is unknown by most clinicians and pathologists in Brazil; (2) establishes the clinical and pathological criteria for the diagnosis of DA toxicosis in dogs; and (3) calls up the attention for the risks of using DA in dogs in clinical settings.
\end{abstract}

INDEX TERMS: Diseases of dogs, toxicosis, toxicology, antiprotozoal therapy, diminazene aceturate.

RESUMO.- Os aspectos epidemiológicos, clínicos e anatomopatológicos da intoxicação espontânea por aceturato de diminazeno foram estudados em 10 cães. Em todos os casos, os cães afetados demonstraram sinais de síndrome tálamo-cortical, principalmente alteração do nível de consciência,

\footnotetext{
${ }^{1}$ Recebido em 17 de dezembro de 2013.

Aceito para publicação em 13 de maio de 2014.

${ }^{2}$ Programa de Pós-Graduação em Medicina Veterinária, área de concentração em Patologia Veterinária, Centro de Ciências Rurais (CCR), Universidade Federal de Santa Maria (UFSM), Camobi, Santa Maria, RS 97105900, Brasil.

${ }^{3}$ Curso de Medicina Veterinária, CCR-UFSM, Santa Maria, RS. Bolsista PIBIC/CNPq/UFSM.

${ }^{4}$ Serviço de Neurologia, Hospital Veterinário Universitário, Departamento de Clínica de Pequenos Animais, CCR-UFSM, Av. Roraima 1000, Santa Maria, RS 97105-900.

${ }^{5}$ Departamento de Patologia, Centro de Ciências da Saúde, UFSM, Av. Roraima 1000, Santa Maria, RS 97105-900. *Autor para correspondência: anemiaveterinaria@yahoo.com.br
}

tetraparesia, rigidez extensora e crise convulsiva. Em alguns casos, os cães acometidos apresentaram sinais de síndrome cerebelar, como tremores musculares generalizados de alta frequência e baixa amplitude, e/ou de síndrome vestibular, como ataxia, inclinação de cabeça e quedas. Esses sinais ocorreram entre 24 e 48 horas após o uso do fármaco injetável por via intramuscular e se mantiveram até a morte ou eutanásia dos cães (entre 1 e 7 dias). Tais sinais clínicos refletiam encefalomalacia hemorrágica focal simétrica, que afetava a medula oblonga, a ponte, a medular do cerebelo, o tálamo, o mesencéfalo, os pedúnculos cerebelares e os núcleos da base. Esse artigo: 1) descreve e discute essa forma de intoxicação medicamentosa tão pouco citada na literatura internacional e desconhecida da maior parte dos clínicos e patologistas veterinários brasileiros, 2) estabelece critérios clínicos e anatomopatológicos para o seu diagnóstico e, principalmente, 3 ) atenta para os riscos da utilização desse princípio ativo na terapêutica canina. 
TERMOS DE INDEXAÇÃO: Doenças de cães, toxicose, toxicologia, terapia antiprotozoário, aceturato de diminazeno.

\section{INTRODUÇÃO}

Aceturato de diminazeno é um medicamento antiprotozoário comumente utilizado em medicina veterinária no tratamento de algumas doenças parasitárias, como babesiose e tripanossomíase (Silva et al. 2008). Para muitos autores, esse quimioterápico é altamente eficaz contra tripanossomas (Egbe-Nwiyi \& Antia 1993) e babésias (Vial \& Gorenflot 2006), que infectam cães. Apesar da maioria dos medicamentos com esse princípio ativo e que estão disponíveis no Brasil ser indicada apenas para bovinos, ovinos, caprinos e equinos, clínicos de pequenos animais frequentemente utilizam tais fármacos como parte de seu arsenal terapêutico. Isso ocorre principalmente porque são reconhecidamente eficazes no tratamento de doenças causadas por hematozoários em cães (Jacobson et al. 1996, Akpa et al. 2008).

Nos Estados Unidos, aceturato de diminazeno tem uso proibido pelo FDA (Food and Drug Administration) (Taboada \& Lobetti 2006), mas, em outros países, principalmente naqueles da África, o fármaco é ainda hoje bastante utilizado no tratamento da babesiose (Collett 2000) e da tripanossomíase (Obi et al. 2013) caninas, duas dentre as causas mais comuns de morte de cães na África do Sul (Collett 2000) e Nigéria (Anene et al. 2006), respectivamente. Nesses dois países, aceturato de diminazeno ainda permanece sendo o agente antiprotozoário mais comumente utilizado (Naidoo et al. 2009), apesar de haver relatos de resistência por determinadas cepas de Babesia gibsoni (Hwang et al. 2010) e Trypanosoma brucei brucei (Anene et al. 2006).

Embora a intoxicação por aceturato de diminazeno tenha sido descrita em cães desde a década de 1970, existem apenas cinco citações sobre essa toxicose na literatura internacional, a saber: 1) um resumo em anais de congresso (Boyt et al. 1968), 2) um relato curto de alguns casos (Losos \& Crockett 1969), 3) um trabalho científico experimental (Naudé et al. 1970), 4) um relato de um caso (van Heerden 1981) e 5) uma descrição acerca de um caso submetido ao seminário semanal (Wednesday Slide Conference) da AFIP (Armed Forces Institute of Pathology) em 2000 (AFIP 2000). No Brasil foram descritos casos na forma de resumos em anais de congressos (Loretti 2002, Pescador et al. 2003, Ferreira et al. 2007, Masuda et al. 2009). Entretanto, apesar de haver pouquíssimos dados na literatura, criadores de cães têm relatado pela internet vários casos de morte após o uso desse fármaco, inclusive no Brasil (Les Amis Samoyedas 2012). Para se ter uma ideia da prevalência dessa forma de intoxicação, o serviço de patologia de um grande instituto de pesquisas veterinárias (Onderstepoort Veterinary Institute), vinculado à maior faculdade de ciências veterinárias da África $(F a-$ culty of Veterinary Sciences, University of Pretoria), teve 21 casos descritos em seus arquivos em um período de apenas três anos (1967-1969) (Pienaar \& Basson 1969, comunicação pessoal).

A falta de informação acerca dessa intoxicação medicamentosa tem feito com que muitos clínicos brasileiros de pequenos animais utilizem o fármaco no tratamento de cães, desconhecendo os possíveis riscos dessa conduta. Assim, o objetivo deste trabalho é apresentar os achados epidemiológicos, clínicos e anatomopatológicos vistos em 10 cães intoxicados após terem sido tratados com medicamentos que tinham como princípio ativo o aceturato de diminazeno, a fim de alertar veterinários em geral, mas principalmente clínicos de pequenos animais, para a ocorrência dessa forma de intoxicação.

\section{MATERIAL E MÉTODOS}

Os casos incluídos neste trabalho ocorreram entre 2008 e 2013 no Município de Santa Maria, localizado na Mesorregião do Centro Ocidental Rio-Grandense, popularmente conhecida como Região Central. Foram considerados apenas casos fatais da doença $(n=10)$. Desses 10 cães, todos foram avaliados clinicamente; cinco foram reavaliados por um neurologista veterinário (A.M.) e posteriormente necropsiados.

Os achados epidemiológicos e os dados clínicos foram fornecidos pelos veterinários responsáveis por cada caso. Os veterinários foram arguidos acerca do princípio ativo das medicações utilizadas, da posologia (dose, via de aplicação e intervalo de utilização), do conhecimento sobre a neurotoxicidade do princípio ativo e da doença que estavam hipoteticamente tratando.

0 exame neurológico foi embasado em Thomas \& Dewey (2003) e Sharp \& Wheeler (2005) e constituído de: 1) observações gerais; 2) avaliação da locomoção (postura e marcha); 3) avaliação das reações posturais; 4) avaliação dos nervos cranianos; 5) avaliação dos reflexos segmentares espinhais; e 6) palpação da coluna vertebral (palpação epaxial).

Vários fragmentos de órgãos foram colhidos à necropsia, fixados em formol tamponado a $10 \%$, processados rotineiramente para histologia e corados pela hematoxilina e eosina (HE). Secções padronizadas do encéfalo e da medula espinhal foram processadas rotineiramente para a preparação de lâminas histológicas a fim de descrever a distribuição e frequência das lesões, conforme estabelecido por Silva et al. (2009). Essas secções incluíam: (1) lobo frontal (ao nível do joelho do corpo caloso [incluindo os núcleos basais]); (2) lobo parietal; (3) lobo temporal; (4) hipocampo; (5) lobo piriforme; (6) diencéfalo; (7) lobo occipital; (8) mesencéfalo (na altura dos colículos rostrais); (9) ponte (com pedúnculos cerebelares); e (10) cerebelo e medula oblonga. A medula espinhal foi dividida em quatro regiões: cervical cranial (C1-C5), cervicotorácica (C6-T2), toracolombar (T3-L3) e lombossacral (L4-S3). A região cervical cranial compreendia os segmentos cervicais craniais e médios; a região cervicotorácica incluía os segmentos cervicais caudais e os torácicos craniais; a região toracolombar compreendia os segmentos torácicos médio-caudais e os lombares craniais e a região lombossacral representava os segmentos lombares caudais e os segmentos sacrais.

0 método de inclusão dos casos neste estudo foi o diagnóstico definitivo de uma lesão neurológica através do exame físico associado ao histórico de uso recente de aceturato de diminazeno.

\section{RESULTADOS}

\section{Epidemiologia}

Dos 10 cães intoxicados por aceturato de diminazeno, cinco $(50 \%)$ eram machos e cinco (50\%) eram fêmeas. A idade dos cães afetados variou de um a três anos. Dos 10 cães afetados, quatro (40\%) não tinha raça definida (SRD) e seis $(60 \%)$ eram de raça pura (RP). As RPs incluíam Pastor Alemão, Akita, Fila Brasileiro, Poodle, Boxer e Samoieda. Dos 10 cães afetados, oito (80\%) morreram espontane- 
amente e dois (20\%) foram submetidos à eutanásia. Sete dos 10 cães afetados (70\%) foram medicados com apenas uma dose do medicamento (3,5 mg/kg de peso vivo). Em três cães afetados (30\%) foram utilizadas duas doses do medicamento (3,5 $\mathrm{mg} / \mathrm{kg}$ de peso vivo) com intervalo de 48 horas, totalizando $7 \mathrm{mg} / \mathrm{kg}$ de peso vivo. Dos 10 clínicos entrevistados, quatro (40\%) não pesaram o cão antes de calcular a dose do medicamento a ser aplicado, ou seja, estipularam o peso apenas visualmente. Seis clínicos relataram ter estabelecido o peso no momento da consulta. Em todos os casos, o início da manifestação clínica ocorreu entre 24 e 48 horas após o uso do aceturato de diminazeno injetável por via intramuscular prescrito por médicos veterinários. Desses, oito eram os mesmos profissionais contatados no momento do diagnóstico. Desses oito colegas, apenas dois (25\%) sabiam sobre a neurotoxicidade do princípio ativo; cinco estavam tratando os cães para o "mal do sangue" (rangeliose canina) e três para babesiose. Dois colegas não eram mais os responsáveis pelo caso e por questões éticas seus nomes não foram revelados e, portanto, eles não puderam ser encontrados para responder as perguntas referentes ao conhecimento da neurotoxicidade e a doença a ser tratada.

\section{Sinais clínicos}

De modo geral, os cães descritos nesse artigo $(n=10)$ demonstraram doença caracterizada invariavelmente apenas por sinais neurológicos. Em nove dos 10 cães afetados (90\%), a evolução clínica foi de 24 a 72 horas. Um dos cães afetados demonstrou sinais neurológicos por sete dias antes de ser submetido à eutanásia in extremis. Os principais sinais clínicos incluíram: alteração do nível de consciência $(10 / 10$ [100\%]), sendo seis casos de estupor (60\%) e quatro casos de sonolência (40\%), tetraparesia (10/10 [100\%]), rigidez extensora por descerebração (7/10 [70\%]), crises convulsivas generalizadas tônico-clônicas (6/10 [60\%]), opistótono (4/10 [40\%]), delírio e vocalização (4/10 [40\%]), e tremores musculares generalizados de alta frequência e baixa amplitude (4/10 [40\%]). Outros sinais clínicos, vistos com menor prevalência, incluíram: ataxia tipo vestibular (2/10 [20\%]), inclinação de cabeça (Fig.1) $(2 / 10$ [20\%]) e quedas $(2 / 10$ [20\%]).

\section{Achados de necropsia}

Na necropsia $(n=5)$, todos os cães $(100 \%)$ apresentavam lesões macroscópicas de maior ou menor intensidade na medular do cerebelo (Fig.2) (4/5 [80\%]), no tálamo (Fig.3) $(4 / 5[80 \%])$, na ponte $(3 / 5[60 \%])$, na medula oblonga (3/5 [60\%]), no mesencéfalo $(2 / 5[40 \%])$, nos pedúnculos cerebelares (Fig.2) (2/5 [40\%]) e/ou nos núcleos basais $(2 / 5$ [40\%]). Essas lesões caracterizavam-se por múltiplos focos, bilateralmente simétricos e irregulares, de avermelhamento (5/5 [100\%]) com (Figs.2 e 3) (3/5 [60\%]) ou sem (Fig.4) (2/5 [40\%]) amolecimento e perda de substância encefálica. Em todos esses casos, a interpretação macroscópica dos patologistas foi de malacia hemorrágica, entretanto, nos dois casos em que não havia amolecimento evidente, um diagnóstico morfológico diferencial de hemorragia intramedular foi também considerado.

\section{Achados histopatológicos}

Na histopatologia $(n=5)$, em todos os casos $(100 \%)$, havia graus variáveis de edema e hemorragia focalmente extensos (Fig.5) que afetavam tanto a substância branca quanto a cinzenta. Essas áreas de hemorragia estavam dispostas ao redor (hemorragia perivascular) (Fig.6) ou próximas aos vasos sanguíneos de parede variavelmente hialinizada (vasculite fibrinoide) (Fig.7) (5/5 [100\%]). Grande quantidade de neutrófilos degenerados era visualizada ao redor dos ou próxima aos vasos sanguíneos afetados, na forma de manguitos perivasculares ou como microabscessos (Fig.8) (3/5 [60\%]), respectivamente. Tais vasos eram, por vezes $(2 / 5[40 \%])$, completa ou parcialmente ocluídos por trombos constituídos de fibrina (Fig.9). Nas áreas em que havia núcleos, neurônios degenerados, caracterizados por dissolução e marginação da substância de Nissl (cromatólise central), e neurônios necróticos, caracterizados por terem citoplasma marcadamente eosinofílico ("neurônios vermelhos") e núcleo picnótico, cariorrético ou em cariólise (Fig.10), eram sempre visualizados (100\%). Tais áreas incluíam principalmente os seguintes núcleos: dentado, fastigial, globoso e emboliforme (cerebelo [4/5; 80\%]); medial e lateral posterior (tálamo [4/5; 80\%]); vestibular e solitário (ponte $[3 / 5 ; 60 \%]$ ); grácil, solitário, cuneiforme e olivares (medula oblonga $[3 / 5 ; 60 \%]$ ); vermelho, oculomotor e trigêmeo (mesencéfalo [2/5; 40\%]); globo pálido, putâmen, amídala (núcleos da base $[2 / 5 ; 40 \%]$ ). Além dos núcleos, muitos tratos também foram afetados (4/5; 80\%), mas principalmente o tegmental e o tecto espinhal. Lesões desses tratos foram mais bem visualizadas no mesencéfalo.

Em todos os casos (100\%) havia variável quantidade, geralmente pequena (4/5 [80\%]) e ocasionalmente grande (1/5 [20\%]), de macrófagos com citoplasma marcadamente espumoso, interpretados como macrófagos que fagocitaram restos neuronais lipídicos (células Gitter) (Fig.11). Ao redor das áreas afetadas, em pelo menos alguns casos $(2 / 5$ [40\%]), havia aumento difuso das células da glia (gliose difusa) e estruturas redondas e marcadamente eosinofílicas (esferoides axonais). Na periferia das áreas afetadas, basicamente em capilares da substância branca adjacente aos núcleos, comumente (4/5 [80\%]) havia manguitos perivasculares constituídos por duas a 10 camadas de células mononucleares, principalmente linfócitos e macrófagos, e menor quantidade de plasmócitos (Fig.12). Nesses casos (4/5 [80\%]), alguns desses vasos tinham endotélio tumefeito (hipertrofia de células endoteliais). Astrócitos reativos, caracterizados por citoplasma abundante e eosinofílico e por núcleo excêntrico (gemistócitos) podiam ser vistos próximos às ou no interior das áreas de malacia em pelo menos alguns casos $(2 / 5[40 \%])$.

\section{DISCUSSÃO}

Os sinais clínicos observados nos 10 cães avaliados e as lesões encontradas nos cinco cães necropsiados são idênticos aos descritos na literatura internacional (Naudé et al. 1970) para intoxicação por aceturato de diminazeno e, associados ao testemunho da utilização desse medicamento por parte dos veterinários responsáveis por cada caso, permitem o diagnóstico definitivo. Esses sinais clínicos e 

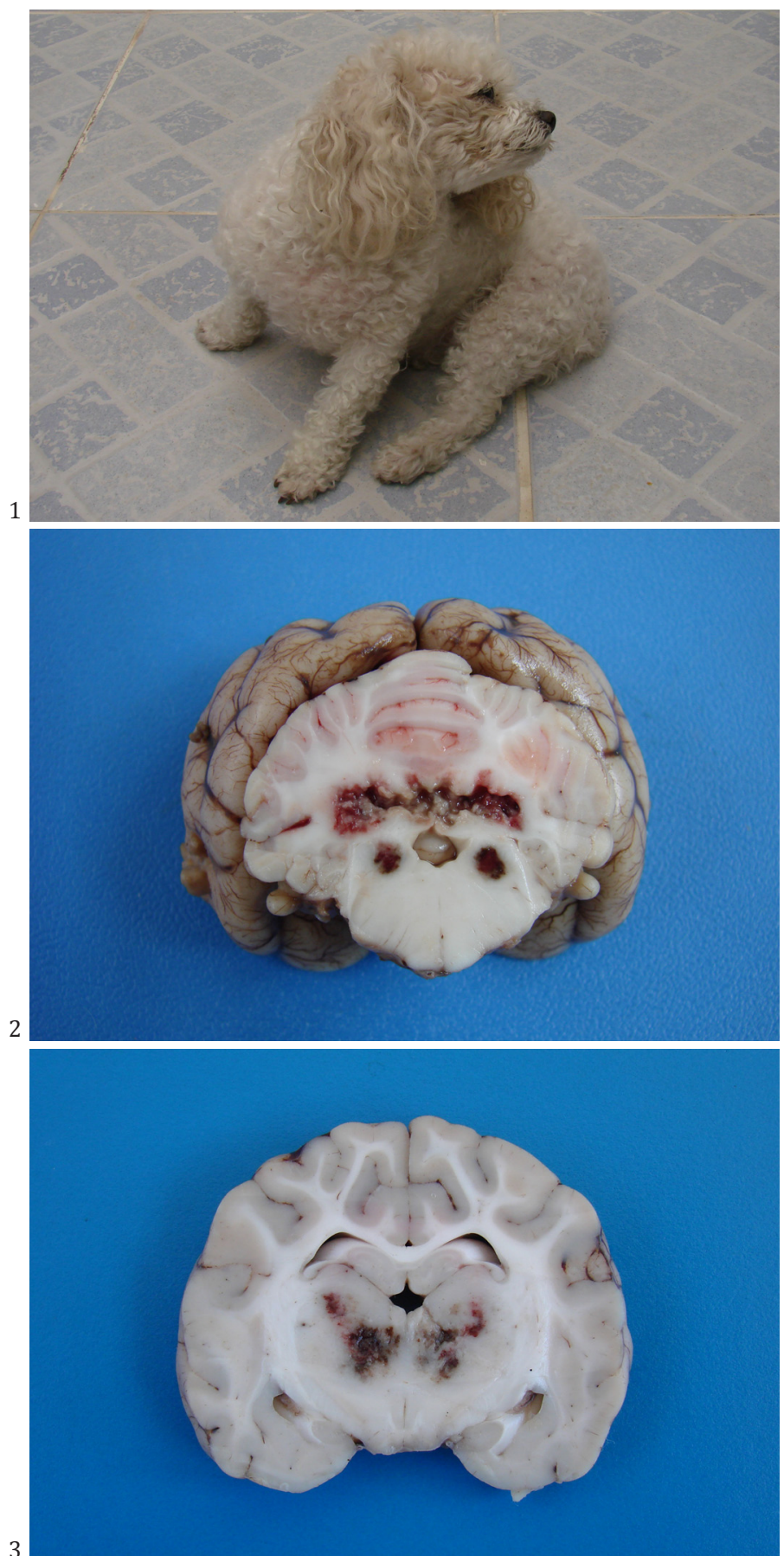

Fig.1. Cão, Poodle. Marcada inclinação de cabeça para o lado esquerdo em um cão intoxicado por aceturato de diminazeno. Esse cão desenvolveu sinais clínicos de síndrome vestibular central que incluíam também ataxia e quedas sempre para o lado esquerdo.

Fig.2. Encéfalo (cerebelo e medula oblonga), superfície de corte (peça fixada em formol). Malacia hemorrágica focalmente extensa da medular do cerebelo e malacia hemorrágica bilateralmente simétrica dos pedúnculos cerebelares caudais em um cão intoxicado por aceturato de diminazeno.

Fig.3. Encéfalo (tálamo [massa intermédia]), superfície de corte (peça fixada em formol). Malacia hemorrágica bilateralmente simétrica em um cão intoxicado por aceturato de diminazeno.
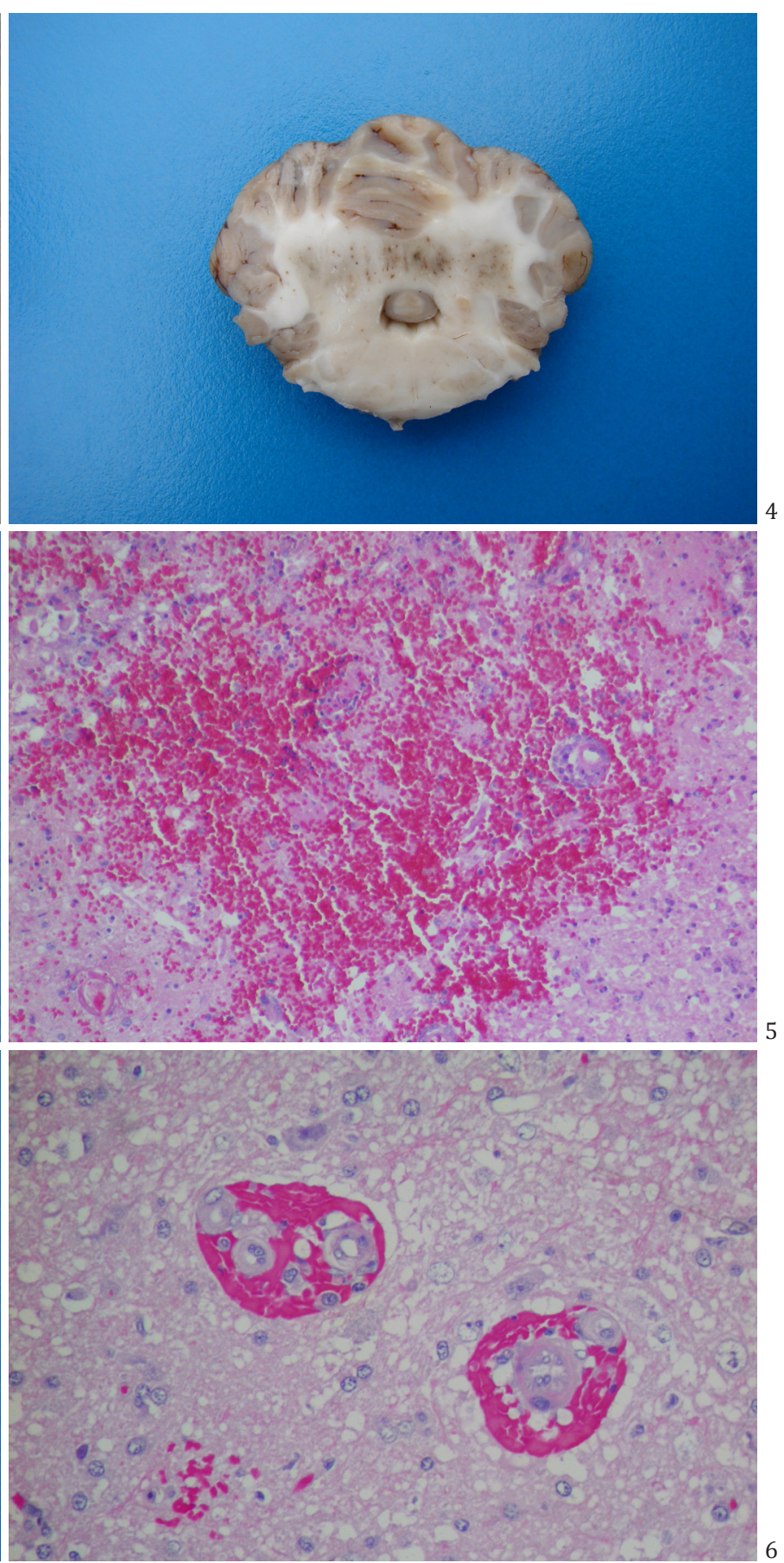

Fig.4. Encéfalo (cerebelo e ponte), superfície de corte (peça fixada em formol). Múltiplas áreas de hemorragia na medular do cerebelo em um cão intoxicado por aceturato de diminazeno. Esse caso histologicamente era idêntico ao da Figura 2, entretanto, a menor gravidade da hemorragia e do edema dificultava um diagnóstico definitivo de malacia hemorrágica apenas pela macroscopia.

Fig.5. Encéfalo. Hemorragia focalmente extensa que obscurece a substância encefálica em um cão intoxicado por aceturato de diminazeno. HE, obj.10x.

Fig.6. Encéfalo. Eritrócitos confinados ao espaço de Virchow-Robin (hemorragia perivascular) e leve vacuolização do neurópilo (edema) em um cão intoxicado por aceturato de diminazeno. HE, obj.20x. 

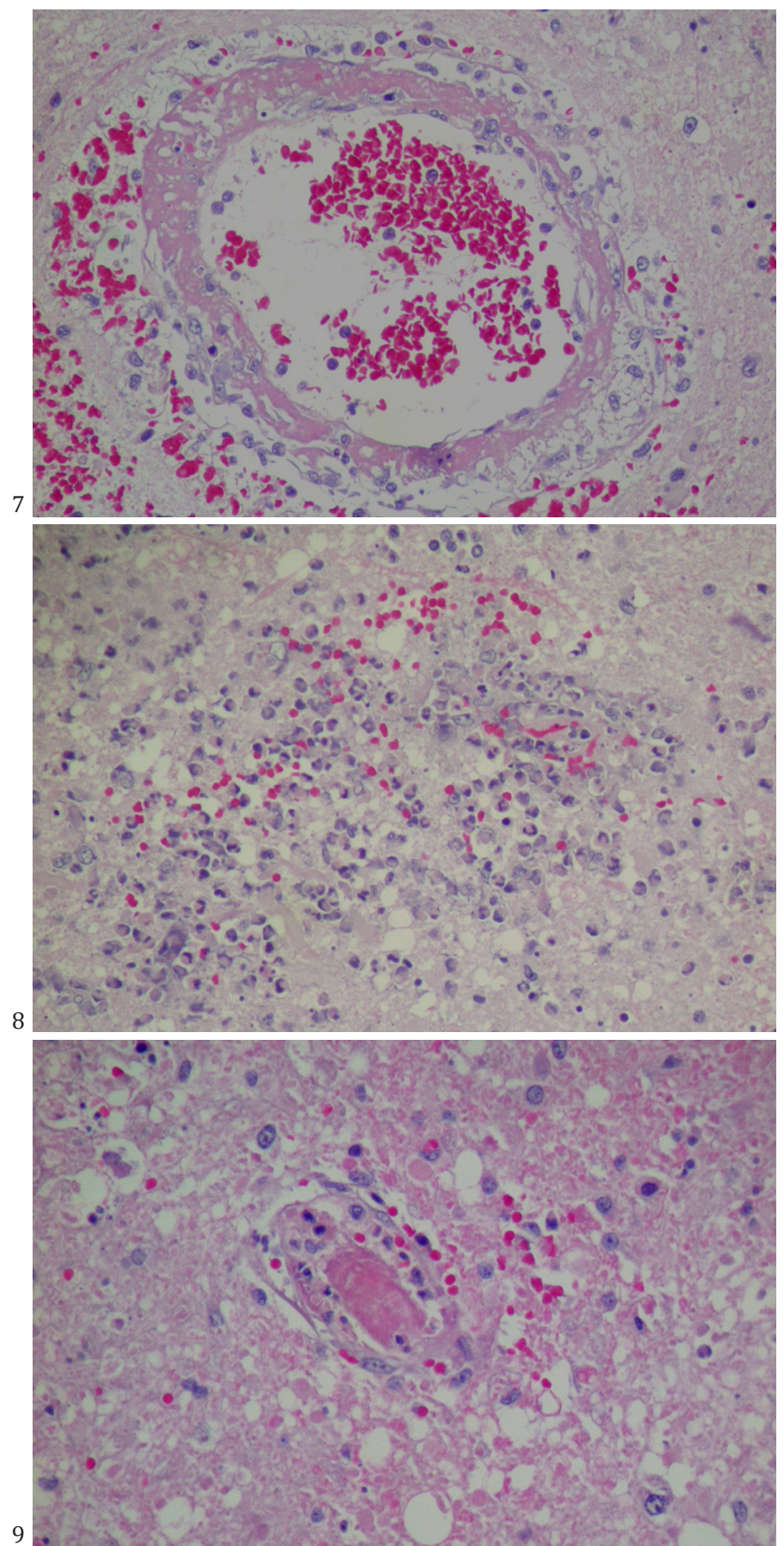

Fig.7. Encéfalo. Vasculite fibrinoide em um cão intoxicado por aceturato de diminazeno. Observe que a parede do vaso sanguíneo está completamente fragmentada, hialina e circundada por pequena quantidade de células inflamatórias. HE, obj.20x

Fig.8. Encéfalo. Marcante acúmulo de neutrófilos degenerados no parênquima encefálico (microabscesso) de um cão intoxicado por aceturato de diminazeno. HE, obj.20x.

Fig.9. Encéfalo. Vaso sanguíneo com lúmen obliterado por trombo de fibrina em um cão intoxicado por aceturato de diminazeno. Note a fina parede do capilar infiltrada por células inflamatórias. HE, obj.20x.
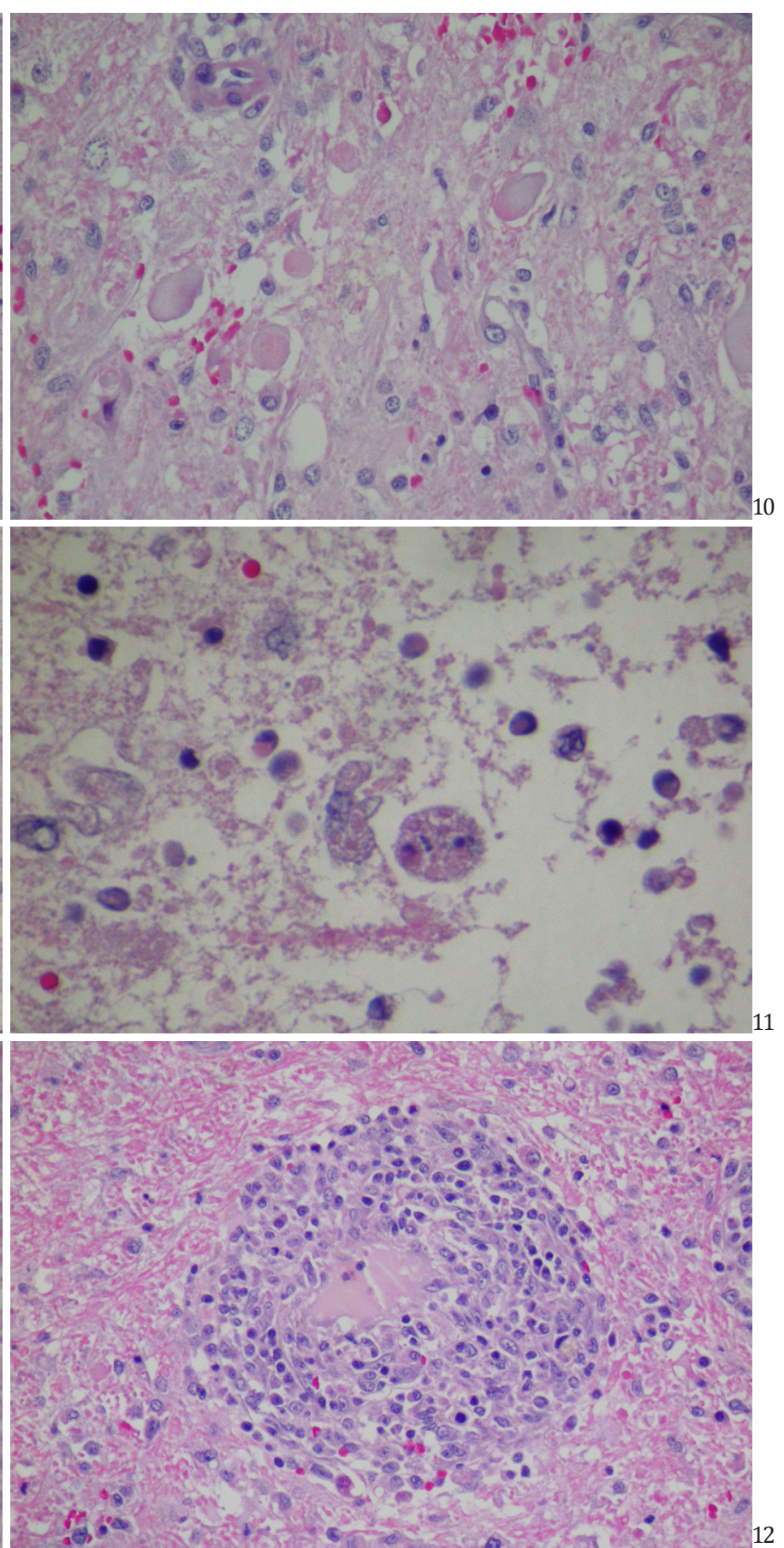

Fig.10. Encéfalo (núcleo). Necrose neuronal em um cão intoxicado por aceturato de diminazeno. HE, obj.20x.

Fig.11. Encéfalo. Rarefação marcada do neurópilo devido a edema e perda de neurônios em um cão intoxicado por aceturato de diminazeno. Note duas células com citoplasma intensamente flocular (células Gitter) no centro do campo. HE, obj.40x.

Fig.12. Encéfalo. Espaço de Virchow-Robin completamente obliterado por células inflamatórias, principalmente macrófagos e linfócitos, com menor quantidade de plasmócitos, em um cão intoxicado por aceturato de diminazeno. Esses manguitos perivasculares são frequentemente vistos ao redor dos núcleos afetados. HE, obj.20x. 
essas lesões foram pela primeira vez relacionados ao uso do aceturato de diminazeno em junho de 1968 (Boyt et al. 1968) em um congresso realizado por uma associação de médicos veterinários da Rodésia (Rhodesian Veterinary Association), atual República do Zimbabwe, mas já eram suspeitados desde o final da década de 1930, quando Lourie \& Yorke (1939) utilizaram diamidinas aromáticas no tratamento de cãezinhos com babesiose por Babesia canis, alguns dos quais acabaram morrendo de uma doença neurológica. Posteriormente, um estudo experimental descreveu com maestria o padrão das lesões e a neurolocalização (Naudé et al. 1970), pondo fim às dúvidas acerca do tema e demonstrando que os sinais clínicos decorriam das extensas áreas de malacia hemorrágica, principalmente no cerebelo (100\% dos casos) (sempre na medular [principalmente afetando os núcleos localizados no teto do quarto ventrículo $\{100 \%$ dos casos $\}$, na medula oblonga $(70 \%)$, na ponte (70\%), no mesencéfalo (60\%) e no tálamo (50\%), mas também, menos frequentemente $(20 \%)$, nos núcleos basais e nos pedúnculos cerebelares. Tais achados anatomopatológicos são muito semelhantes aos descritos nesses cinco cães necropsiados, tanto qualitativa como quantitativamente.

Os achados clínicos e anatomopatológicos observados nesses casos de intoxicação por aceturato de diminazeno permitem inferir que o medicamento causa lesão vascular, que afeta especificamente capilares encefálicos, basicamente aqueles que irrigam o tronco encefálico e a porção medular do cerebelo. Essa lesão possivelmente desencadeia menor aporte sanguíneo à substância cinzenta, devido à trombose subsequente ao dano endotelial, o que culmina em hipoxia neuronal e, consequentemente, degeneração e necrose dos neurônios dos núcleos da medula oblonga, da ponte, do cerebelo, do tálamo, do mesencéfalo e dos núcleos da base. Trombose secundária à lesão vascular foi citada por Naudé et al. 1970, mas foi dada pouca ênfase a essa patogênese.

Apesar da literatura não estabelecer mecanismo algum para a ocorrência das lesões vasculares encefálicas, experimentos demonstraram que não ocorre alteração da pressão sanguínea (Joubert et al. 2003) nem inibição ou depressão de colinesterases (Milner et al. 1997) quando da utilização de aceturato de diminazeno em cães. Um aspecto interessante observado em trabalhos científicos anteriores realizados com cães infectados experimentalmente por Trypanosoma brucei brucei é que a infecção por esse protozoário retarda marcadamente a eliminação corporal do aceturato de diminazeno (Anika \& Onyeyili 1989) e aumenta seus níveis no encéfalo (Onyeyili \& Anika 1991), o que talvez explique a predileção por afetar vasos sanguíneos apenas dessa região. Resultados semelhantes foram também obtidos com infecção experimental por T. congolense em cães (Onyeyili \& Anika 1989). Esse retardo na eliminação do medicamento, confirmado no final da década de 1980, já era suspeitado há alguns anos, como se pode notar pelo comentário final do relato de caso de van Heerden (1981), em que ele ressalta um aumento na suscetibilidade do cão com babesiose em desenvolver intoxicação, mesmo utilizando a dose terapêutica do medicamento. Uma teoria antiga, já ci- tada por Naudé et al. (1970), e relacionada com efeitos semelhantes ao da histamina, que aumenta a permeabilidade vascular, é normalmente reproduzida por outros autores (AFIP 2000) como uma possibilidade de patogênese.

Nos casos em que o cão morreu ou foi submetido à eutanásia tão cedo quanto 24 a 48 horas do início dos sinais clínicos, as lesões macroscópicas foram escassas e caracterizaram-se basicamente por avermelhamento leve das áreas afetadas, como pode ser visto na Fig.4. Nos casos em que o cão morreu ou foi submetido à eutanásia após 48 horas do início dos sinais clínicos, as lesões macroscópicas foram marcantes e tipicamente refletiam uma encefalomalacia hemorrágica simétrica, como pode ser visto nas Figuras 2 e 3. Histologicamente, lesões mais agudas apresentaram vasculite fibrinoide, rarefação do neurópilo, devido ao edema, e hemorragia perivascular. Nas lesões mais avançadas observou-se hemorragia disseminada que obliterava os núcleos e espalhava-se pela substância branca e um infiltrado inflamatório massivo de neutrófilos na forma de microabscessos. Essa sequência de lesões observadas nos casos descritos é muito semelhante àquelas vistas por Naudé et al. (1970) em seu experimento pioneiro acerca desse tema. Independentemente do momento da morte, todos os encéfalos dos cães avaliados tinham lesões suficientemente graves para explicar os sinais clínicos apresentados.

Quanto aos sinais clínicos, a maior parte dos cães (60\%) desenvolveu manifestação caracterizada por alteração do nível de consciência (sonolência ou estupor), tetraparesia, rigidez extensora por descerebração e crises convulsivas generalizadas tônico-clônicas. Além disso, quase a metade dos cães $(40 \%)$ desenvolveu opistótono, delírio e vocalização. Tais sinais são típicos de síndrome tálamo-cortical (Fenner 2004). Nenhum desses cães demonstrou histologicamente lesão de córtex telencefálico, mas todos tinham lesão na massa intermédia do tálamo $(60 \%)$ ou nos núcleos da base (40\%). Portanto, pode ser afirmado que a lesão responsável pelos sinais clínicos vistos nos cães avaliados clinica e anatomopatologicamente teve origem diencefálica ou nos núcleos da base.

Pelo menos $40 \%$ dos cães afetados apresentaram tremores musculares generalizados de alta frequência e baixa amplitude, sinal clínico típico de síndrome cerebelar. Nenhum desses cães demonstrou histologicamente lesão de córtex cerebelar, mas todos tinham lesão na medular do cerebelo. Portanto, a lesão responsável pelos sinais clínicos vistos em $40 \%$ dos cães avaliados clínica e anatomopatologicamente teve origem cerebelar, mais especificamente nos núcleos dentado, globoso e emboliforme, pois lesões no núcleo fastigial causam síndrome vestibular central (Sanders \& Bagley 2003).

Uma menor parte dos cães (20\%) desenvolveu manifestação caracterizada por ataxia tipo vestibular, inclinação de cabeça e quedas. Tais lesões são típicas de síndrome vestibular. Esses cães demonstraram lesões histológicas na medula oblonga, na ponte, na medular do cerebelo, nos pedúnculos cerebelares e no mesencéfalo, entretanto, apenas um desses cães $(10 \%)$ desenvolveu sinais cerebelares (tremores musculares generalizados de alta frequência e baixa amplitude). Isso ocorre porque a manifestação clíni- 
ca predominante em casos de lesão de núcleos da medular do cerebelo não necessariamente é de síndrome cerebelar, mas pode sim ser de síndrome vestibular central. A explicação para isso está no fato de que alguns axônios vestibulares desviam dos núcleos do tronco encefálico através dos pedúnculos cerebelares caudais e fazem sinapse no núcleo fastigial do cerebelo, o que faz com que uma lesão nesse núcleo e nesses pedúnculos, como visto em $80 \%$ e $40 \%$ dos casos aqui descritos, respectivamente, manifeste-se como síndrome vestibular central e não como síndrome cerebelar (Sanders \& Bagley 2003). Portanto, pode se afirmar que a lesão responsável pelos sinais clínicos vistos nessa minoria dos cães avaliados clinica e anatomopatologicamente teve origem na medula oblonga, na ponte, na medular do cerebelo e nos pedúnculos cerebelares.

Cabe comentar que, apesar de ter havido uma prevalência um pouco maior de lesão na medula oblonga, na ponte e na medular do cerebelo do que no tálamo e nos núcleos da base, os sinais clínicos de síndrome tálamo-cortical foram cinco vezes mais comuns do que aqueles de síndrome vestibular e duas vezes mais comuns do que aqueles de síndrome cerebelar. As hipóteses para isso incluem: 1) em casos de lesões multifocais poderá haver predomínio de uma das manifestações, o que pode dificultar o diagnóstico clínico das áreas realmente afetadas (Bagley 2004), 2) a lesão que afetou o tronco encefálico e a medular do cerebelo foi sempre bilateral e sabe-se que em casos de síndrome vestibular central decorrente de lesões bilaterais podem não ocorrer alguns sinais clássicos, como nistagmo e inclinação de cabeça (Sanders \& Bagley 2003), e/ou 3) o fato de todos os cães chegarem para o atendimento com nível de consciência alterado, sendo $60 \%$ deles em estupor, pode ter mascarado muito o exame neurológico, dificultado sobremaneira o reconhecimento dos sinais típicos de síndrome vestibular.

Todos os cães descritos receberam medicamentos à base de aceturato de diminazeno em doses terapêuticas (3,5 $\mathrm{mg} / \mathrm{kg}$ de peso vivo), conforme estabelecido na literatura médica veterinária internacional (Taboada \& Lobetti 2006). Desses, três utilizaram o fármaco por duas vezes, o que é considerado contra-indicado (Miller et al. 2005). Entretanto, pelo menos quatro clínicos admitiram que não pesaram os cães e que o peso utilizado no cálculo da dose foi estipulado apenas visualmente. Isso nos leva a presumir que: 1) pelo menos três cães (30\% dos casos) intoxicaram-se devido à reutilização do fármaco, 2) quatro cães ( $40 \%$ dos casos) intoxicaram-se por terem seu peso corporal possivelmente superestimado, o que aumentou a dose total do medicamento aplicado e 3) pelo menos três cães (30\% dos casos) intoxicaram-se realmente com a dose terapêutica do produto (provável reação idiossincrásica). No único estudo experimental realizado a fim de estabelecer se realmente ocorreria intoxicação pelo aceturato de diminazeno em cães concluiu-se que, devido ao medicamento ser potencialmente tóxico, sua dosagem terapêutica jamais deve ser ultrapassada e sua utilização não pode ser repetida no curso da doença (Naudé et al. 1970). Além disso, Van Heerden (1981) descreveu um caso de intoxicação em que a superdosagem decorreu da somatória entre o aceturato de diminazeno e a fenamidina, chamando a atenção para essa interação medicamentosa fatal. De qualquer forma, os autores (Naudé et al. 1970, Van Heerden 1981) são unânimes em afirmar que há uma marcada variação individual na suscetibilidade de cães em desenvolver reação ao uso desse princípio ativo. Além disso, casos com sinais clínicos e lesões características à necropsia já foram associados à dosagem terapêutica, bem como outros foram associados a erros na concentração do princípio ativo expressa no rótulo do produto (AFIP 2000).

Outro dado interessante é que, apesar dos clínicos entrevistados terem conhecimento sobre o fato do aceturato de diminazeno ser um fármaco de índice terapêutico reconhecidamente baixo (Peregrine \& Mamman 1993), apenas $25 \%$ conhecia a associação entre seu uso e doença neurológica (neurotoxicidade). Tais colegas afirmam utilizar medicamentos que contêm o princípio ativo e não terem tido, até então, problemas de reações adversas. Esse é um ponto interessante, pois se sabe que não são todos os cães tratados que desenvolvem lesões encefálicas e, consequentemente, sinais clínicos.

\section{CONCLUSÕES}

Os resultados apresentados demonstram a ocorrência de casos de intoxicação por aceturato de diminazeno em cães na Região Central do Rio Grande do Sul (10 casos em cinco anos) e o desconhecimento da maioria dos veterinários acerca da neurotoxicidade desse princípio ativo. Assim, os clínicos de pequenos animais e os patologistas veterinários devem considerar a possibilidade de intoxicação diante de históricos de doença neurológica aguda em cães com suspeita ou diagnóstico definitivo de babesiose ou rangeliose, pois esse é o perfil dos pacientes que fazem uso de medicações antiprotozoários na Região Central do Estado.

Clinicamente, o diagnóstico de intoxicação por aceturato de diminazeno deverá ser aventado sempre que ocorrerem sinais de síndrome tálamo-cortical, síndrome cerebelar e/ ou de síndrome vestibular central em cães que fizeram uso recente do medicamento (24-48 horas anteriores ao início dos sinais clínicos). Esse diagnóstico deverá ser aventado toda vez em que houver encefalomalacia hemorrágica bilateralmente simétrica que afeta o tronco encefálico e, ocasionalmente, estende-se para os núcleos da base e para a medular do cerebelo, em cães que fizeram uso recente do medicamento.

Com base nos achados desse estudo observa-se que a intoxicação por aceturato de diminazeno ocorre principalmente quando a dose considerada terapêutica é excedida como, por exemplo, quando o peso do cão é superestimado ou o clínico resolve utilizar uma segunda aplicação do medicamento, mas também em casos em que a dose utilizada é aquela recomendada na literatura. Dessa forma, é importante ressaltar que, embora muitos clínicos possam utilizar esse fármaco rotineiramente na dosagem indicada na bula e que os casos de intoxicação sejam vistos apenas ocasionalmente, não existe como saber previamente se determinado cão desenvolverá ou não lesão vascular encefálica após sua utilização. Portanto, a única forma segura de 
prevenir a ocorrência dessa intoxicação é não utilizar esse princípio ativo como parte do arsenal terapêutico da clínica médica canina.

Finalmente, recomenda-se a inclusão de informações acerca da possibilidade de lesão vascular encefálica e consequentes sinais clínicos neurológicos em cães, incluindo a ocorrência de morte, na bula de medicamentos que contenham aceturato de diminazeno, independentemente de serem indicados para cães ou apenas para grandes animais.

\section{REFERÊNCIAS}

AFIP 2000. Armed Forces Institute of Pathology Wednesday Slide Conference, 18:4. Disponível em <http://www.askjpc.org/wsco/wsc/wsc99/ 99wsc18.htm> Acesso em 22 set. 2013.

Akpa P.O., Ezeokonkwo R.C., Eze C.A. \& Anene B.M. 2008. Comparative efficacy assessment of pentamidine isethionate and diminazene aceturate in the chemotherapy of Trypanosoma brucei brucei infection in dogs. Vet. Parasitol. 151:139-149.

Anene B.M., Ezeokonkwo R.C., Mmesirionye T.I., Tettey J.N., Brock J.M., Barret M.P. \& De Koning H.P. 2006. A diminazene-resistant strain of Trypanosoma brucei brucei isolated from a dog is cross-resistant to pentamidine in experimentally infected albino rats. Parasitol. 132:127-133.

Anika S.M. \& Onyeyili P.A. 1989. Effects of trypanosomal infection on the pharmacokinetics of diminazene aceturate in dogs. Trop. Med. Parasitol. 40:419-421.

Bagley R.S. 2004. Doença neurológica multifocal, p.638-644. In: Ettinger S.J. \& Feldman E.C. (Eds), Tratado de Medicina Interna Veterinária: doenças do cão e do gato. $5^{\text {a }}$ ed. Guanabara Koogan, Rio de Janeiro. 2156p.

Boyt W.P., Lawrence J.A. \& Mackenzie P.K.I. 1968. The toxicity of berenil. Anais de Rhodesian Veterinary Conference. Rhodesian Veterinary Conference, Rhodesia, p.33. (Resumo)

Collett M.G. 2000. Survey of canine babesiosis in South Africa. J. South Afr. Vet. Assoc. 71:180-186.

Egbe-Nwiyi T.N. \& Antia R.E. 1993. The effect of trypanocidal drug treatment on the haematological changes in Trypanosoma brucei brucei infected splenectomised dogs. Vet. Parasitol. 50:23-33.

Fenner W.R. 2004. Doenças do cérebro, p.586-638. In: Ettinger S.J. \& Feldman E.C. (Eds), Tratado de Medicina Interna Veterinária: doenças do cão e do gato. $5^{\underline{a}}$ ed. Guanabara Koogan, Rio de Janeiro. 2156p.

Ferreira E.V., Boabaid F.M., Gasparetto N.D., Fontana D.S., Oliveira P.M., Silva I.S., Souza M.A., Nakazato L. \& Colodel E.M. 2007. Achados clínicos e patológicos na intoxicação por diaceturato de diminazeno em dois cães. Anais do XIII Encontro Nacional de Patologia Veterinária (Enapave), Campo Grande, MS, s/p. (Resumo)

Hwang S.J., Yamasaki M., Nakamura K., Murakami M., Wickramasekara Rajapakshage B.K., Ohta H., Maede Y. \& Takiguchi M. 2010. Development and characterization of a strain of Babesia gibsoni resistant to diminazene aceturate in vitro. J. Vet. Med. Sci. 72:765-777.

Jacobson L.S., Reyers F., Berry W.L. \& Viljoen E. 1996. Changes in haematocrit after treatment of uncomplicated canine babesiosis: a comparison between diminazene and trypan blue, and an evaluation of the influence of parasitaemia. J. South Afr. Vet. Assoc. 67:77-82.

Joubert K.E., Kettner F., Lobetti R.G. \& Miller D.M. 2003. The effects of diminazene aceturate on systemic blood pressure in clinically healthy adult dogs. J. South Afr. Vet. Assoc. 74:69-71.

Les Amis Samoyedas 2012. Medicação tóxica para Samoiedas. Disponível em: http:// http://www.canillesamis.com.br/saude/medicacao-toxica-para-samoieda. Acesso em 22 set. 2013.

Loretti A.P. 2002. Focal symmetrical hemorrhagic encephalomalacia as- sociated with diminazene aceturate therapy in a dog. Anais III Reunión Argentina de Patologia Veterinaria (Rapave), Rosario, Santa Fe, Argentina, p.54. (Resumo)

Losos G.J. \& Crockett E. 1969. Toxicity of beril in the dog. Vet. Rec. 85:196.

Lourie E.M. \& Yorke W. 1939. Studies in chemotherapy. XXII. The action of certain aromatic diamidines on Babesia canis infections of puppies. Ann. Trop. Med. Parasitol. 33:305-312.

Masuda E.K., Sant'Ana F.J.F., Tessele B. \& Kommers G.D. 2009. Intoxicação em cães por aceturato de diminazeno. Anais XIV Encontro Nacional de Patologia Veterinária (Enapave), Águas de Lindóia, São Paulo, Brasil, s/p. (Resumo)

Miller D.M., Swan G.E., Lobetti R.G. \& Jacobson L.S. 2005. The pharmacokinetics of diminazene aceturate after intramuscular administration in healthy dogs. J. South Afr. Vet. Assoc. 76:146-150.

Milner R.J., Reyers F., Taylor J.H. \& Van den Berg J.S. 1997. The effect of diminazene aceturate on cholinesterase activity in dogs with canine babesiosis. J. South Afr. Vet. Assoc. 68:111-113.

Naidoo V., Mulders M.S. \& Swan G.E. 2009. The intravenous pharmacokinetics of diminazene in healthy dogs. J. South Afr. Vet. Assoc. 80:215-219.

Naudé T.W., Basson P.A. \& Pienaar J.G. 1970. Experimental diamidine poisoning due to commonly used babecides. Onderstepoort J. Vet. Res. 37:173-184.

Obi C.F., Obidike R.I., Ezeh I.O., Omoja V.U., Iheagwam C.N., Idika I.K. \& Ezeokonkwo R.C. 2013. Effects of Trypanosoma brucei infection and diminazene aceturate therapy on testicular morphology and function of Nigerian local dogs. Vet. Parasitol. 196:283-288.

Onyeyili P.A. \& Anika S.M. 1989. The influence of Trypanosoma congolense infection on the disposition kinetics of diminazene aceturate in the dog. Vet. Res. Commun. 13:231-236.

Onyeyili P.A. \& Anika S.M. 1991. Diminazene aceturate residues in the tissues of healthy, Trypanosoma congolense and Trypanosoma brucei brucei infected dogs. Brit. Vet. J. 147:155-162.

Peregrine A.S. \& Mamman M. 1993. Pharmacology of diminazene: a review. Acta Trop. 54:185-203.

Pescador C.A., Loretti A.P. \& Both A.C. 2003. Intoxicação por aceturato de diminazeno em um canino: relato de caso. Anais XI Encontro Nacional de Patologia Veterinária (Enapave), Botucatu, SP, p.62. (Resumo)

Pienaar J.G. \& Basson P.A. 1969. Comunicação pessoal (Onderstepoort Veterinary Institute, Pretoria, Gauteng, South Africa).

Sanders S.G. \& Bagley R.S. 2003. Disorders of hearing and balance: The vestibulocochlear nerve (CN VIII) and associated structures, p.213-240. In: Dewey C.W. (Ed.), A Pratical Guide to Canine and Feline Neurology. Blackwell, Ames. 642p.

Sharp N.J.H. \& Wheeler S.J. 2005. Small animal spinal disorders: diagnosis and surgery. $2^{\text {nd }}$ ed. Elsevier Mosby, Philadelphia.

Silva A.S., Tochetto C., Zanette R.A., Pierezan F., Rissi D.R., Santurio J.M. \& Monteiro S.G. 2008. Aceturato de diminazeno e dipropionato de imidocarb no controle de infecção por Trypanossoma evansi em Rattus norvegicus infectados experimentalmente. Ciência Rural 38:1357-1362.

Silva M.C., Fighera R.A., Mazzanti A., Brum J.S., Pierezan F. \& Barros C.S.L. 2009. Neuropatologia da cinomose canina. Pesq. Vet. Bras. 29:643-652.

Taboada J. \& Lobetti R. 2006. Babesiosis, p.722-736. In: Greene C.E. (Ed.), Infectious Diseases of the Dog and Cat. $3^{\text {rd }}$ ed. Elsevier, St Louis. 1387p.

Thomas W.B. \& Dewey C.W. 2003. Performing the neurologic examination, p.31-55. In: Dewey C.W. (Ed.), A Pratical Guide to Canine and Feline Neurology. Blackwell, Ames. 642p.

Van Heerden J. 1981. Diamidine poisoning in a dog. J. South Afr. Vet. Assoc. 52:338-339.

Vial H.J. \& Gorenflot A. 2006. Chemotherapy against babesiosis. Vet. Parasitol. 138:147-160. 